\title{
Synaptonemal Complex Components Are Required for Meiotic Checkpoint Function in Caenorhabditis elegans
}

\author{
Tisha Bohr, Guinevere Ashley, ${ }^{1}$ Evan Eggleston, ${ }^{1}$ Kyra Firestone, and Needhi Bhalla ${ }^{2}$ \\ Department of Molecular, Cell, and Developmental Biology, University of California, Santa Cruz, California 95064
}

\begin{abstract}
Synapsis involves the assembly of a proteinaceous structure, the synaptonemal complex (SC), between paired homologous chromosomes, and is essential for proper meiotic chromosome segregation. In Caenorhabditis elegans, the synapsis checkpoint selectively removes nuclei with unsynapsed chromosomes by inducing apoptosis. This checkpoint depends on pairing centers (PCs), cis-acting sites that promote pairing and synapsis. We have hypothesized that the stability of homolog pairing at PCs is monitored by this checkpoint. Here, we report that SC components SYP-3, HTP-3, HIM-3, and HTP-1 are required for a functional synapsis checkpoint. Mutation of these components does not abolish PC function, demonstrating they are bona fide checkpoint components. Further, we identify mutant backgrounds in which the instability of homolog pairing at PCs does not correlate with the synapsis checkpoint response. Altogether, these data suggest that, in addition to homolog pairing, SC assembly may be monitored by the synapsis checkpoint.
\end{abstract}

KEYWORDS meiosis; synapsis; chromosome; checkpoint; synaptonemal complex

EIOSIS is the specialized cell division by which cells undergo one round of DNA duplication and two successive rounds of division to produce haploid gametes from diploid organisms. During sexual reproduction, fertilization restores diploidy in the resulting embryo. In order for meiotic chromosomes to segregate properly in meiosis I and II, homologs pair, synapse, and undergo crossover recombination (Bhalla et al. 2008). If homologous chromosomes fail to segregate properly, this can produce gametes, such as egg and sperm, with an improper number of chromosomes, termed aneuploidy. Embryos that result from fertilization of aneuploid gametes are generally inviable, but can also exhibit developmental disorders (Hassold and Hunt 2001). Therefore, checkpoint mechanisms monitor early meiotic prophase events to avoid the production of aneuploid gametes (MacQueen and Hochwagen 2011).

Copyright (C) 2016 by the Genetics Society of America

doi: 10.1534/genetics.116.191494

Manuscript received May 11, 2016; accepted for publication August 30, 2016 ; published Early Online September 6, 2016.

Supplemental material is available online at www.genetics.org/lookup/suppl/doi:10. 1534/genetics.116.191494/-/DC1.

${ }^{1}$ Joint second authors.

${ }^{2}$ Corresponding author: Department of Molecular, Cell, and Developmental Biology, 225 Sinsheimer Labs, University of California, Santa Cruz, CA 95064. E-mail: nbhalla@ ucsc.edu
Synapsis involves the assembly of a proteinaceous complex, the synaptonemal complex (SC), between paired homologous chromosomes, and is essential for crossover recombination (Bhalla and Dernburg 2008). In Caenorhabditis elegans, the synapsis checkpoint induces apoptosis to remove nuclei with unsynapsed chromosomes and prevent aneuploid gametes (Bhalla and Dernburg 2005) (Figure 1A). The synapsis checkpoint requires pairing centers (PCs) (Bhalla and Dernburg 2005), cis-acting sites near one end of each chromosome. PCs also promote pairing and synapsis (MacQueen et al. 2005) by recruiting factors, such as the zinc-fingercontaining proteins ZIM-1, ZIM-2, ZIM-3, and HIM-8 (Phillips et al. 2005; Phillips and Dernburg 2006), and the conserved polo-like kinase PLK-2 (Harper et al. 2011; Labella et al. 2011). We have hypothesized that the synapsis checkpoint monitors the stability of pairing at PCs as a proxy for proper synapsis (Deshong et al. 2014; Bohr et al. 2015). However, whether the process of synapsis is also monitored by the synapsis checkpoint is currently unknown.

Upon entry into meiosis, axial elements assemble between replicated sister chromatids to support homolog pairing and synapsis. In most species, HORMA domain proteins (HORMADs) associate with axial elements (Hollingsworth et al. 1990; Caryl et al. 2000; Wojtasz et al. 2009; Fukuda et al. 

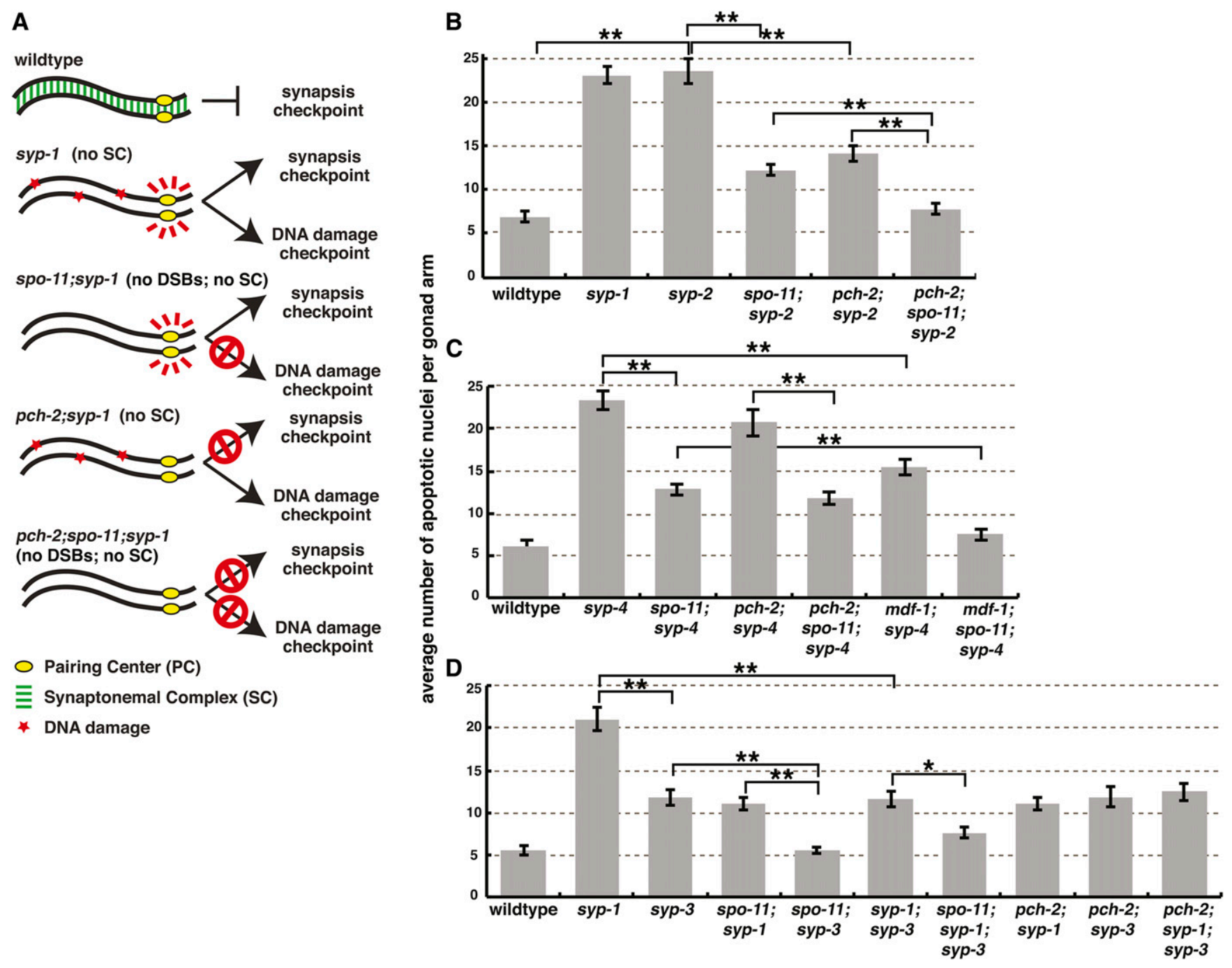

Figure 1 SYP-3 is required for the meiotic synapsis checkpoint. (A) Cartoons depicting meiotic checkpoint activation in C. elegans. (B) Elevation of germline apoptosis in syp-2 mutants is dependent on spo-11 and pch-2. (C) Elevation of germline apoptosis in syp-4 mutants is dependent on spo-11 and $m d f-1$ but not on pch-2. (D) Elevation of germline apoptosis in syp-3 mutants is dependent on spo-11 but not on pch-2. Mutation of syp-3 reduces apoptosis in syp-1 and syp-1; spo-11 double mutants but not syp-1; pch-2 double mutants. Error bars represent \pm SEM. $* P<0.01, * * P<0.0001$.

2010). These proteins share structural features with the wellcharacterized spindle checkpoint protein, Mad2 (Aravind and Koonin 1998; Kim et al. 2014), and have been implicated in monitoring meiotic prophase events, such as recombination and synapsis (Carballo et al. 2008; Daniel et al. 2011; Wojtasz et al. 2012), thus coupling meiotic chromosome architecture to checkpoint function. In C. elegans, four HORMAD proteins, HTP-3, HIM-3, HTP-1, and HTP-2, comprise the axial elements of the SC and play overlapping but distinct roles during meiotic prophase, including but not limited to meiotic checkpoint function (Zetka et al. 1999; Couteau et al. 2004; Couteau and Zetka 2005; Martinez-Perez and Villeneuve 2005; Goodyer et al. 2008; Kim et al. 2015).

Synapsis is complete when the central element of the SC is assembled between paired axial elements of homologous chromosomes. In C. elegans, the central element includes the factors SYP-1, SYP-2, SYP-3, and SYP-4 (MacQueen et al. 2002; Colaiacovo et al. 2003; Smolikov et al. 2007, 2009). Loss of any one of these proteins produces a similar mutant phenotype: extensive asynapsis of all chromosomes that is accompanied by a delay in meiotic progression in which chromosomes remain asymmetrically localized in meiotic nuclei (MacQueen et al. 2002; Colaiacovo et al. 2003; Smolikov et al. 2007, 2009) and factors that normally localize transiently to meiotic chromosomes persist (Harper et al. 2011; Labella et al. 2011; Rosu et al. 2013; Stamper et al. 2013; Woglar et al. 2013). We have shown that syp-1 mutants also induce germline apoptosis as a result of the synapsis checkpoint (Figure 1A) (Bhalla and Dernburg 2005). However, it is unclear whether syp-2, syp-3, or syp-4 mutants similarly elicit an increase in germline apoptosis in response to the synapsis checkpoint. Genetically ablating the synapsis checkpoint does not affect the meiotic delay associated with asynapsis in syp-1 mutants (Deshong et al. 2014; Bohr et al. 
2015), indicating that these two events are not mechanistically coupled. Meiotic HORMAD proteins regulate this delay (Martinez-Perez and Villeneuve 2005; Kim et al. 2015).

Here, we report that some SC components are required for the synapsis checkpoint. syp- 2 mutants resemble syp- $1 \mathrm{mu}-$ tants and elevate apoptosis in response to the synapsis checkpoint. syp-4 mutants also exhibit elevated apoptosis similar to syp-1 and syp-2 mutants. However, the elevation in apoptosis observed in syp-4 mutants is not dependent on PCH-2 but is dependent on MDF-1. Since both PCH-2 and MDF-1 are synapsis checkpoint components (Bhalla and Dernburg 2005; Bohr et al. 2015) that act redundantly to regulate synapsis (Bohr et al. 2015), these data suggest there may be molecular differences in how the synapsis checkpoint can be activated. By contrast, syp-3 mutants do not elicit a synapsis checkpoint response, indicating that SYP-3 is required for the synapsis checkpoint. Similarly, htp-3, him-3, and htp-1 mutants are also defective in the synapsis checkpoint. The ability to generate a synapsis checkpoint response does not correlate with less stable homolog pairing at PCs, suggesting that the synapsis checkpoint may instead monitor SC assembly through these factors. Finally, loss of SYP-3, HTP-3, HIM-3, or HTP-1 does not abrogate PC function, consistent with these proteins playing a direct role in the checkpoint.

\section{Results and Discussion}

\section{SYP-3 is required for the synapsis checkpoint}

syp-1 mutants exhibit increased germline apoptosis as a result of the synapsis checkpoint (due to asynapsis) and the DNA damage checkpoint [due to an inability to repair double strand breaks (DSBs)] (Figure 1A) (Bhalla and Dernburg 2005). SPO-11 is required for the introduction of meiotic DSBs (Dernburg et al. 1998) and PCH-2 is required for the synapsis checkpoint (Bhalla and Dernburg 2005). We have previously shown that loss of SPO-11 or PCH-2 in otherwise wild-type backgrounds does not affect germline apoptosis (Bhalla and Dernburg 2005). However, spo-11; syp-1 and pch-2; syp-1 double mutants display lower levels of germline apoptosis than syp-1 single mutants because of loss of the DNA damage or synapsis checkpoint response, respectively (Figure 1A) (Bhalla and Dernburg 2005). Loss of both checkpoints in pch-2; spo-11; syp-1 triple mutants results in wild-type levels of apoptosis (Figure 1A) (Bhalla and Dernburg 2005).

To determine whether other syp mutants behave similarly, we quantified apoptosis in null syp-2, syp-3, and syp-4 mutants (Figure 1, B-D). Mutation of syp-2 elevated germline apoptosis levels similar to those seen in syp-1 mutants (Figure 1B), suggesting that syp-2 mutants exhibit both DNA damage and synapsis checkpoint responses. To verify that syp-2 mutants exhibit a DNA damage checkpoint response, we introduced a mutation of spo-11 into a syp-2 background. We observed decreased apoptosis to intermediate levels in spo11; syp-2 double mutants (Figure 1B), indicating that syp-2 mutants exhibit a DNA damage checkpoint response. To determine whether syp-2 mutants exhibit a synapsis checkpoint response, we observed apoptosis in pch-2; syp-2 double mutants that also had intermediate levels of germline apoptosis (Figure 1B). This verifies that syp-2 mutants elevate germline apoptosis due to the synapsis checkpoint. Furthermore, mutation of both pch-2 and spo-11 reduced apoptosis to wild-type levels in a syp-2 background (Figure 1B). These data show that the elevation of apoptosis observed in syp-2 mutants is in response to both the DNA damage and synapsis checkpoints, similar to syp-1 mutants (Bhalla and Dernburg 2005).

Next we analyzed syp-4 mutants and found that germline apoptosis was also elevated (Figure 1C) comparable to syp-1 and syp-2 mutants (Figure 1B). Moreover, spo-11; syp-4 double mutants resembled spo-11; syp-1 and spo-11; syp-2 double mutants (Bhalla and Dernburg 2005) (Figure 1, B and C), demonstrating that syp-4 mutants have elevated apoptosis due to the DNA damage checkpoint. However, germline apoptosis was unaffected in pch-2; syp-4 and pch-2; spo-11; syp-4 mutants compared to syp-4 and spo-11; syp-4 mutants, respectively (Figure 1C).

We reasoned that these results with the pch-2 mutation could either reflect that an additional meiotic checkpoint was active in syp-4 mutants or syp-4 mutants produced a synapsis checkpoint response independent of PCH-2. We distinguished between these two possibilities by monitoring germline apoptosis in $m d f-1$; syp-4 double mutants and $m d f-1$; spo-11; syp-4; triple mutants (Figure 1C). We previously reported that MDF-1, the C. elegans ortholog of the spindle checkpoint gene Mad1, is also required for the synapsis checkpoint and regulates synapsis in an independent, parallel pathway to PCH-2 (Bohr et al. 2015). Loss of MDF-1 reduced apoptosis to intermediate levels in syp-4 mutants and wildtype levels in spo-11; syp-4 mutants, indicating that the synapsis checkpoint contributes to the increase in apoptosis observed in syp-4 mutants (Figure 1C). Thus, the genetic requirements for the synapsis checkpoint in syp-4 mutants are different than that of syp-1 and syp-2 mutants.

We also quantified apoptosis in syp-3 mutants and observed increased apoptosis compared to wild-type worms but not to levels observed in syp-1 single mutants (Figure 1D). This suggests that unlike syp-1, syp-2, and syp-4 mutants, syp-3 mutants either have a functional DNA damage or synapsis checkpoint, but not both. To determine which checkpoint was responsible for the elevated apoptosis observed in syp-3 mutants, we first quantified apoptosis in spo-11; syp-3 double mutants (Figure 1D). Mutation of spo-11 in a syp-3 background reduced apoptosis to wild-type levels (Figure 1D), demonstrating that the elevation in apoptosis observed in syp-3 mutants is dependent on the DNA damage checkpoint. To ensure that the elevation in apoptosis observed in syp-3 mutants is due solely to the DNA damage checkpoint and not due to the synapsis checkpoint, we monitored germline apoptosis in pch-2; syp-3 mutants. Mutation of pch-2 in the syp-3 background did not reduce apoptosis 
(Figure 1D), illustrating that the elevation in apoptosis observed in syp-3 mutants is not dependent on the synapsis checkpoint. Therefore, although chromosomes are unsynapsed in syp-3 mutants (Smolikov et al. 2007), the synapsis checkpoint response is abrogated.

These data suggest that SYP-3 is required for the synapsis checkpoint. To verify this, we quantified apoptosis in syp-1; syp-3 double mutants (Figure 1D). syp-1; syp-3 double mutants had intermediate levels of germline apoptosis (Figure 1D), indicating loss of either the DNA damage checkpoint or the synapsis checkpoint but not both. Mutation of syp-3 in a pch-2; syp-1 background did not further decrease apoptosis (Figure 1D), confirming that SYP-3 is not required for the DNA damage checkpoint. However, spo-11; syp-1; syp-3 triple mutants had wild-type levels of apoptosis (Figure 1D), signifying loss of the synapsis checkpoint. Altogether these data show that SYP-3, but not SYP-2 or SYP-4, is required for the synapsis checkpoint.

\section{syp-3 and syp-4 mutants exhibit more stable PC pairing than syp-1 mutants}

In the absence of synapsis (for example, in syp-1 mutants), we can visualize pairing intermediates that typically precede and promote synapsis (MacQueen et al. 2002). Loss of PCH-2 further stabilizes pairing in syp-1 mutants (Deshong et al. 2014), leading us to hypothesize that this stabilization of pairing, particularly at PCs, satisfies the synapsis checkpoint in pch-2; syp-1 and pch-2; syp-2 double mutants. We reasoned that since syp-3 and syp-4 mutants behaved differently than syp-1 and syp-2 mutants in the context of checkpoint activation, there might be similar differences with respect to PC pairing. We monitored pairing of $X$ chromosomes as a function of meiotic progression by performing immunofluorescence against the PC protein HIM-8 (Phillips et al. 2005) in syp-1, syp-3, and syp-4 mutants, both in the presence and absence of PCH-2 (Figure 2A). Meiotic nuclei are arrayed in a spatiotemporal gradient in the germline, allowing for the analysis of the progression of meiotic events as a function of position in the germline (Figure 2B, see cartoon). We divided the germline into six equivalently sized zones and assessed the number of nuclei with paired HIM-8 signals in each zone. All six strains initiated pairing in zone 2, achieved maximal pairing by zone 4 , and destabilized pairing in zones 5 and 6 (Figure 2B). Although we observed that loss of $\mathrm{PCH}-2$ had effects on pairing in zone 6 in both syp-3 and syp-4 mutants (Figure 2B), signifying a role for $\mathrm{PCH}-2$ in these backgrounds independent of the synapsis checkpoint, we focused our analysis on zone 2, based on the more stable pairing we detected in pch-2; syp-1 double mutants in comparison to syp-1 single mutants in this region (Figure 2B). PCs were more frequently paired in both syp-3 and syp-4 single mutants, similar to pch-2; syp-1 mutants, in zone 2. pch-2; syp-3 double mutants exhibited less steady-state pairing at $X$ chromosome PCs than syp-3 single mutants in zone 2, suggesting that in this background PCH-2 somehow promotes stable PC pairing. pch-2; syp-4 double mutants resembled syp-4 single mutants in zone 2 , indicating that loss of PCH-2 in syp-4 mutants does not further stabilize pairing at PCs and providing a potential explanation for why PCH-2 is not required for the synapsis checkpoint in syp-4 mutants. Further, since syp-4 mutants present similar frequencies of stable homolog pairing at PCs as pch-2; syp-1 double mutants and nonetheless elicit a synapsis checkpoint response (Figure 1C) while pch-2; syp-3 double mutants have paired PCs as infrequently as syp-1 single mutants and do not activate germline apoptosis via the synapsis checkpoint (Figure 1D), these results suggest that stable PC pairing cannot be the sole criteria that satisfies the synapsis checkpoint.

\section{HORMAD proteins HTP-3, HIM-3, and HTP-1 are required for the synapsis checkpoint}

We also tested whether axial element proteins, specifically HORMADs, are required for the synapsis checkpoint using null mutations of each gene (Figure 3). First, we tested whether HTP-3 and HIM-3 are required for the synapsis checkpoint by monitoring apoptosis in htp-3 and him-3 mutants (Figure 3A). htp-3 and him-3 mutants produced wild-type levels of apoptosis (Figure 3A), despite their inability to synapse chromosomes (Zetka et al. 1999; Couteau et al. 2004; Goodyer et al. 2008). Thus, these mutants produce neither a DNA damage checkpoint nor a synapsis checkpoint response. HTP-3 is required for DSB formation in meiosis (Goodyer et al. 2008) and HIM-3 is thought to promote interhomolog recombination by inhibiting intersister repair (Couteau et al. 2004). These phenotypes could explain the inability of these mutants to generate a DNA damage response. To further investigate a possible role for HTP-3 and HIM-3 in the synapsis checkpoint, we introduced mutations of $h t p-3$ and him-3 into syp-1 mutants and quantified apoptosis. syp-1; $h t p-3$ and syp-1; him-3 double mutants have wild-type levels of germline apoptosis (Figure 3A), demonstrating that, even in the syp-1 background, HTP-3 and HIM-3 are indeed required for the synapsis checkpoint.

We then tested whether HTP-1 and HTP-2 are required for the synapsis checkpoint. $h t p-1$ single mutants synapse their chromosomes nonhomologously (Couteau and Zetka 2005; Martinez-Perez and Villeneuve 2005) and had intermediate levels of apoptosis (Figure 3B). These data suggest that $h t p-1$ mutants elicit a DNA damage or synapsis checkpoint response but not both. $h t p-2$ single mutants have no obvious meiotic defects (Couteau and Zetka 2005) and exhibited wild-type levels of apoptosis (Figure 3B), indicating that $h t p-2$ mutants do not produce a DNA damage or synapsis checkpoint response. $h t p-1$ is linked to spo-11 on chromosome IV, making it difficult to create spo-11 htp-1 double mutants. Therefore, to investigate which checkpoint was responsible for the intermediate levels of apoptosis observed in $h t p-1$ mutants, we abrogated the DNA damage checkpoint using a mutation in cep-1, the $C$. elegans p53 ortholog (Derry et al. 2001; Schumacher et al. 2001). Mutation of cep-1 in the $h t p-1$ background reduced apoptosis to wild-type levels while mutations of pch-2 had no effect on 

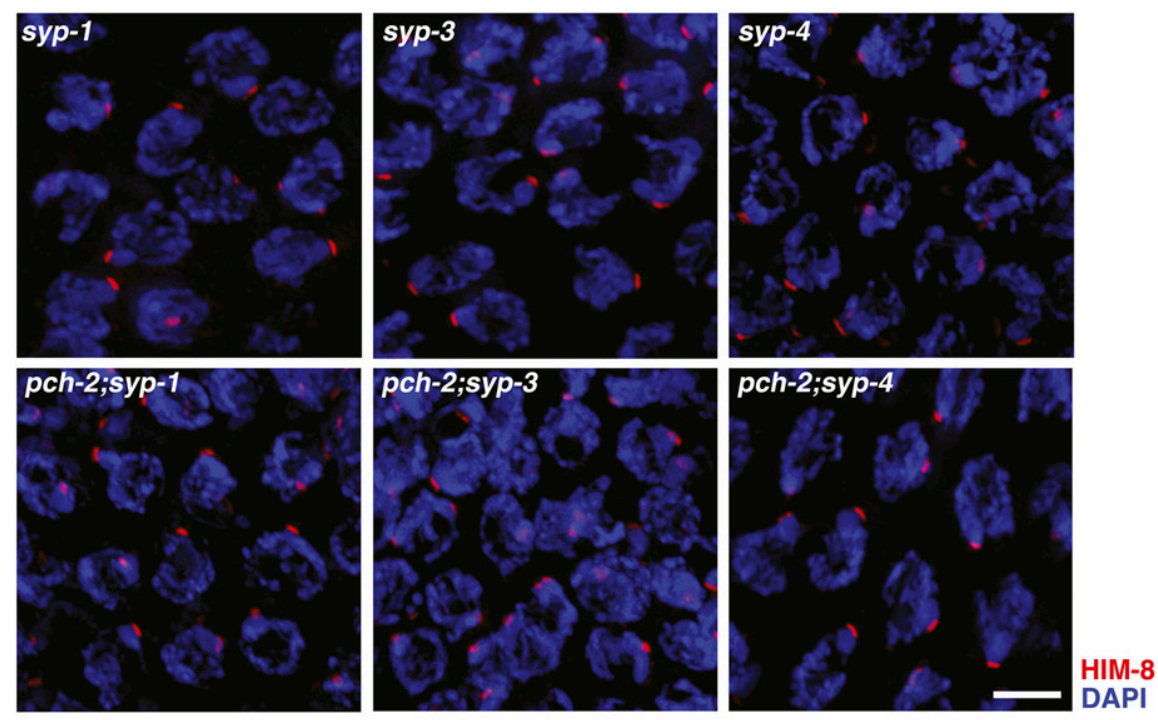

\section{B}

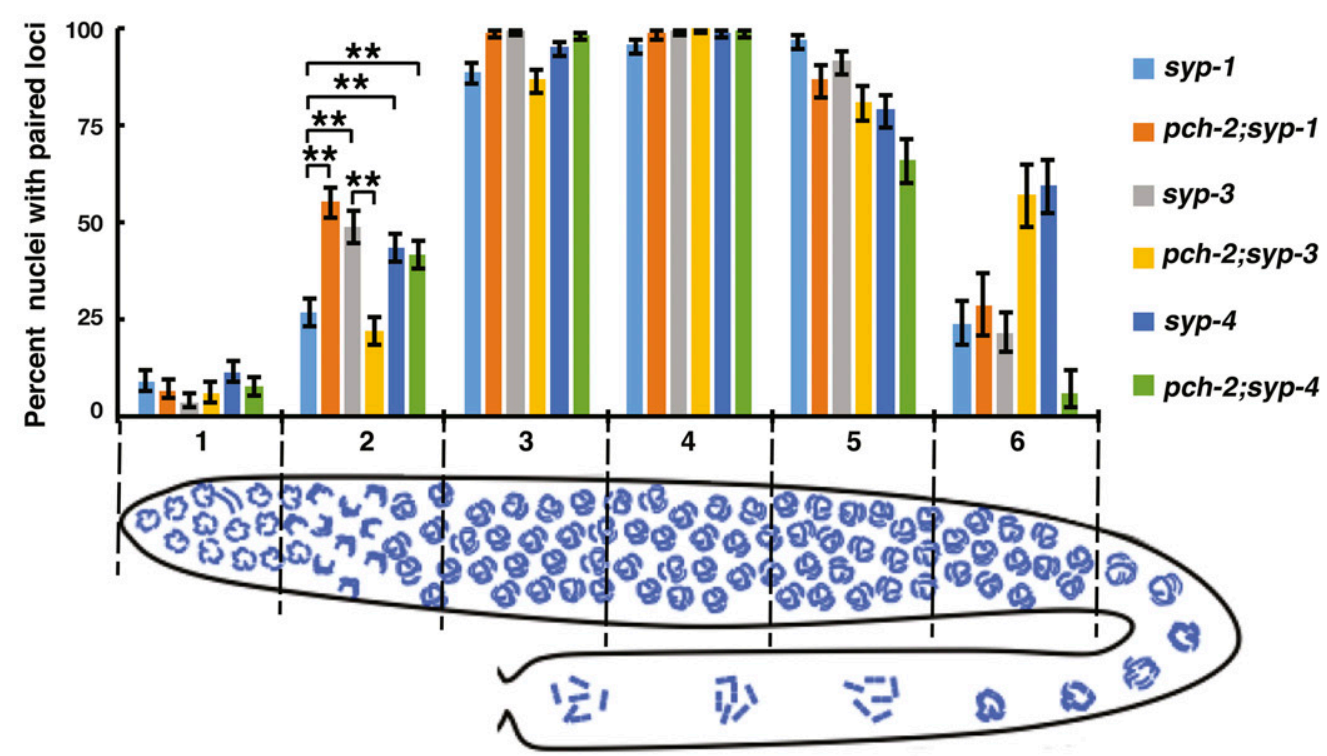

Figure 2 syp-3 and syp-4 mutants exhibit more stable $P C$ pairing than syp-1 mutants. (A) Images of meiotic nuclei in syp-1, pch-2; syp-1, syp-3, pch-2; syp-3, syp-4, and pch-2; syp4 mutants stained to visualize HIM-8 (red) and DNA (blue). Bars, $5 \mu \mathrm{m}$. (B) Pairing at the $X$ chromosome PC is more stable in syp-3, syp-4, and pch-2; syp-4 mutants than in syp- 1 mutants. The numbers on the $x$-axis correspond to regions of the gonad depicted in the cartoon in B. Meiotic progression is from left to right. Error bars represent $95 \%$ confidence intervals. ** $P<0.0001$. Significance was assessed by performing Fisher's exact test.

germline apoptosis when compared to $h t p-1$ single mutants (Figure 3B). This indicates that the elevation in apoptosis observed in $h t p-1$ mutants is dependent on the DNA damage checkpoint and not the synapsis checkpoint. Therefore, unlike $h t p-3$ and him-3 mutants (Figure 3A), htp-1 mutants activate germline apoptosis in response to the DNA damage checkpoint (Figure 3B), supporting the idea that meiotic HORMADS also play distinct roles during meiotic checkpoint activation. Furthermore, these data suggest that either nonhomologous synapsis does not result in a synapsis checkpoint response or that HTP-1 may be required for the synapsis checkpoint.

To test whether HTP-1 is required for the synapsis checkpoint, we took advantage of the partially redundant roles of
HTP-1 and HTP-2 during meiotic synapsis. htp-1 htp-2 double mutants have unsynapsed chromosomes (Couteau and Zetka 2005), similar to htp-3 and him-3 single mutants (Zetka et al. 1999; Couteau et al. 2004; Goodyer et al. 2008), allowing us to evaluate whether unsynapsed chromosomes elicit a synapsis checkpoint response in the absence of HTP-1. Similar to htp-1 single mutants, $h t p-1$ $h t p-2$ double mutants exhibited intermediate apoptosis (Figure 3B), suggesting that abrogation of the synapsis checkpoint in $h t p-1$ mutants is not the product of nonhomologous synapsis and supporting the possibility that HTP- 1 is required for the synapsis checkpoint. In addition, these data demonstrate that HTP-1 and HTP-2 do not appear to play redundant roles in the DNA damage checkpoint's induction 

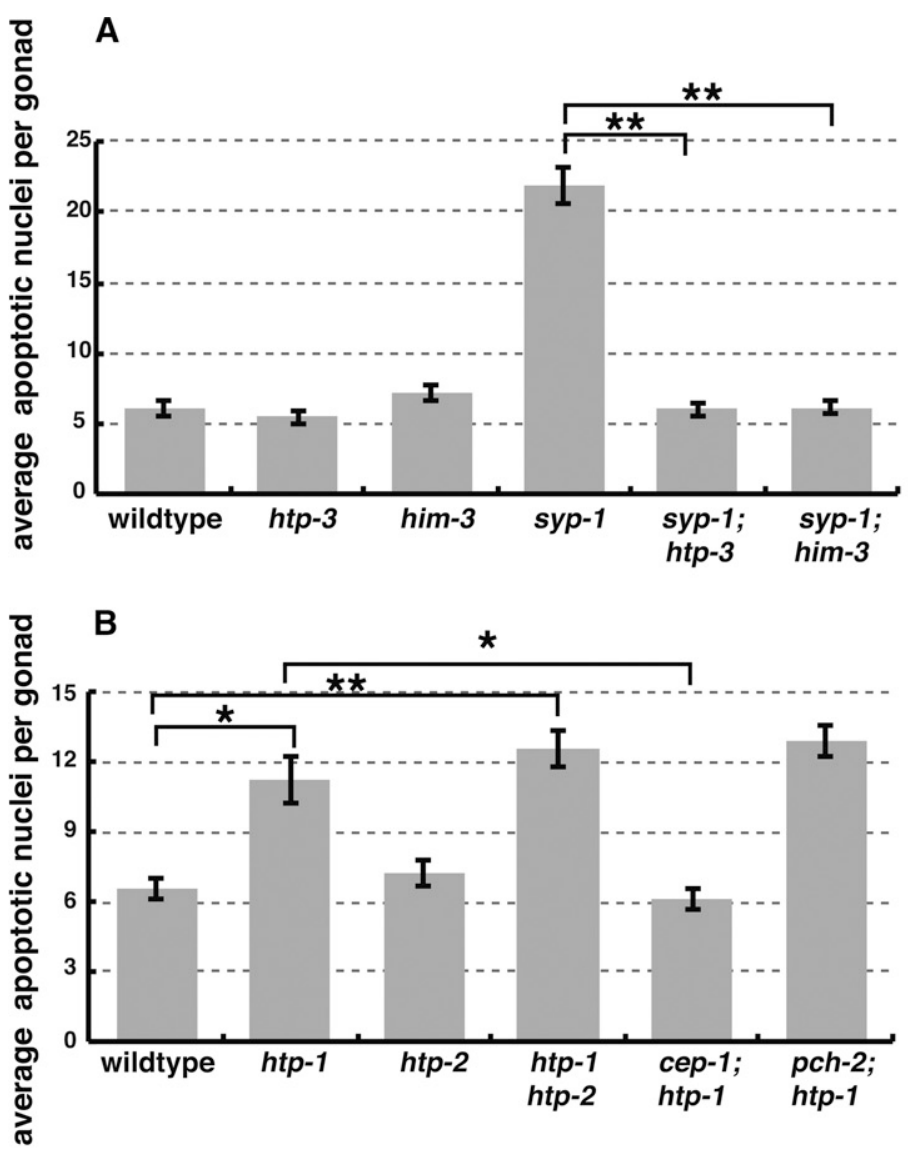

Figure 3 HTP-3, HIM-3, and HTP-1 are required for the synapsis checkpoint. (A) htp-3 and him-3 mutants have wild-type levels of germline apoptosis and reduce germline apoptosis in syp-1 mutants. (B) The elevation of germline apoptosis in htp-1 mutants is cep-1 dependent but not pch-2 dependent. (C) Mutation of $h t p-1$ reduces germline apoptosis in syp-1 single and cep-1; syp-1 double mutants. Error bars represent \pm SEM. * $P<0.01, * *$ $P<0.0001$

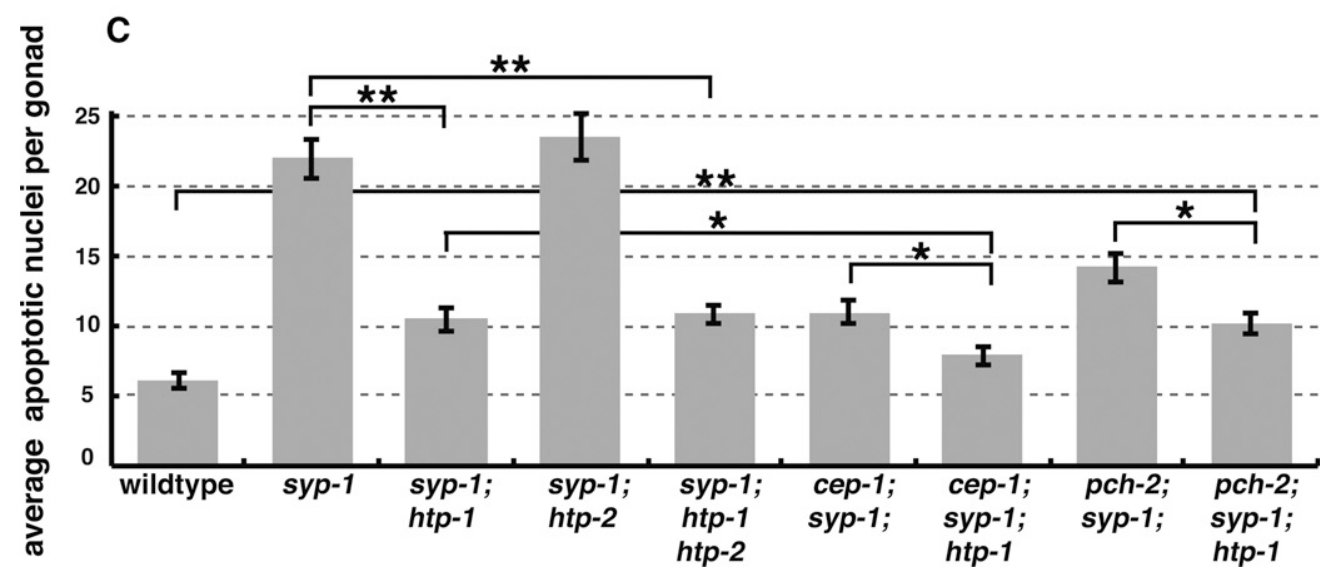

of germline apoptosis. This is in contrast to the redundant roles they play in regulating meiotic progression when chromosomes are unsynapsed (Kim et al. 2015).

To validate that HTP- 1 is required for the synapsis checkpoint, we observed apoptosis in syp- $1 ; h t p-1$ and syp- $1 ; h t p-2$ double mutants (Figure 3C). While mutation of $h t p-2$ had no effect on apoptosis in the syp-1 background, we observed reduced apoptosis to intermediate levels in syp- 1 ; $h t p-1$ double mutants compared to syp-1 single mutants (Figure 3C), indicating loss of one checkpoint. To verify that the synapsis checkpoint but not the DNA damage checkpoint is abrogated in the syp-1; $h t p-1$ background we observed apoptosis in pch-2; syp-1; htp-1 and cep-1; syp-1; htp-1 triple mutants. Mutation of cep-1 in the syp-1; $h$ tp-1 background reduced apoptosis to levels comparable to wild-type worms (Figure 3C), demonstrating that the elevation of apoptosis observed in syp-1; $h t p-1$ mutants is dependent on the DNA damage checkpoint. In addition, mutation of pch-2 did not further decrease apoptosis in the syp-1; $h t p-1$ background (Figure 3C), showing that the elevation of apoptosis observed in 
A
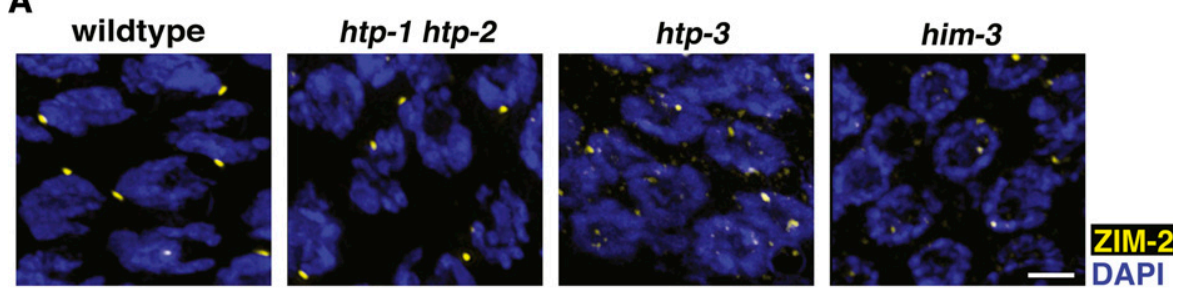

B
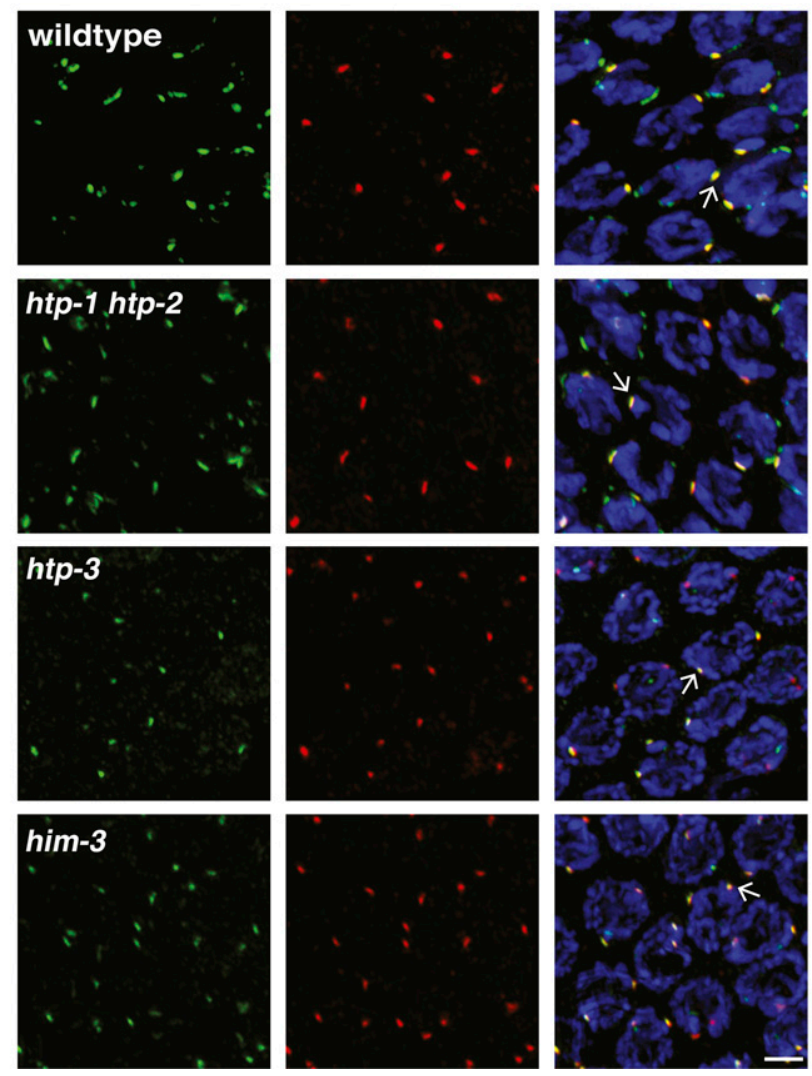

Figure 4 Loss of HTP-3 and HIM-3 disrupts localization of some but not all PC proteins. (A) Images of early meiotic prophase nuclei in wildtype worms, htp-1/2, htp-3, and him-3 mutants stained to visualize ZIM-2 (yellow) and DAPI (blue). (B) Images of early meiotic prophase nuclei in wild-type worms, $h t p-1 / 2$, htp-3, and, him-3 mutants stained to visualize PLK-2 (green), HIM-8 (red), and DAPI (blue). Arrow indicates an example of colocalization of PLK2 and HIM-8. Bar, $2 \mu \mathrm{m}$. syp- 1 ; $h t p-1$ mutants is not dependent on the synapsis checkpoint. Therefore, the synapsis checkpoint is abrogated in syp-1; htp-1 mutants. However, while apoptosis in pch-2; syp-1; $h t p-1$ triple mutants was significantly higher than wild-type, pch-2; syp-1; htp-1 triple mutants had reduced levels of apoptosis in comparison to pch-2; syp-1 double mutants (Figure 3C), suggesting that loss of HTP-1 affects the synapsis checkpoint more severely than loss of PCH-2. Alternatively, loss of HTP-1 may partially reduce the DNA damage response in this background, given its role in enforcing meiotic-specific DNA repair mechanisms (Martinez-Perez and Villeneuve 2005). Lastly, similar to syp-1; htp-1 double mutants, syp-1; htp-1 htp-2 triple mutants exhibited intermediate levels of apoptosis compared to syp-1 single mutants and wild-type worms (Figure 3C), further verifying that HTP-2 is not redundant with HTP-1 when considering checkpoint activation of apoptosis. Altogether, these data illustrate that HTP-3, HIM-3, and HTP-1, but not HTP-2, are required for the synapsis checkpoint.

\section{HTP-3 and HIM-3 disrupt localization of some but not all PC proteins}

HTP-3, HIM-3, and HTP-1 could be directly required for the synapsis checkpoint or these proteins could be involved in regulating other mechanisms that are required for the synapsis checkpoint. For example, since PCs are required for the synapsis checkpoint (Bhalla and Dernburg 2005), we were concerned that $h t p-3$, him-3, and $h t p-1$ mutants might have defects in PC function. Since $h t p-1$ single mutants produce nonhomologous synapsis (Couteau and Zetka 2005; Martinez-Perez and Villeneuve 2005) and our analysis of apoptosis shows that loss of HTP-2 has no effect on synapsis checkpoint signaling (Figure 3C), we performed experiments to address this using $h t p-1 h t p-2$ double mutants, which have unsynapsed chromosomes (Couteau and Zetka 2005), allowing better comparison with $h t p-3$ and him-3 single mutants. We localized ZIM-2, a protein that binds to and is required for PC function of chromosome V (Phillips and Dernburg 2006), in wild-type worms and htp-3, him-3, and htp-1 htp-2 
A

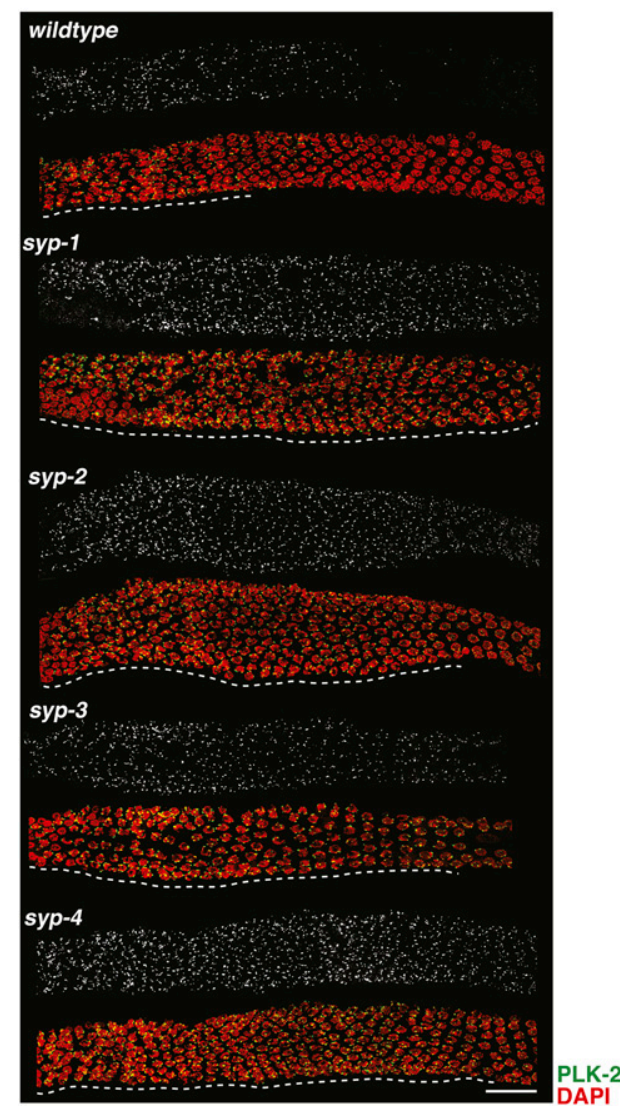

B

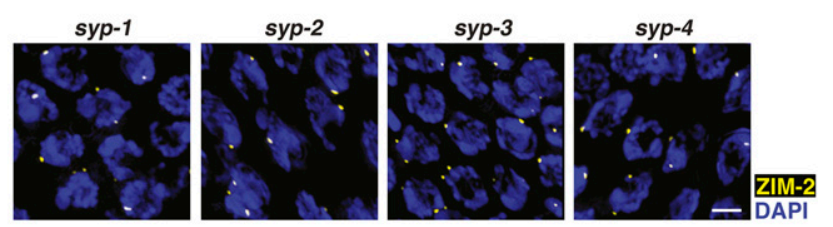

C
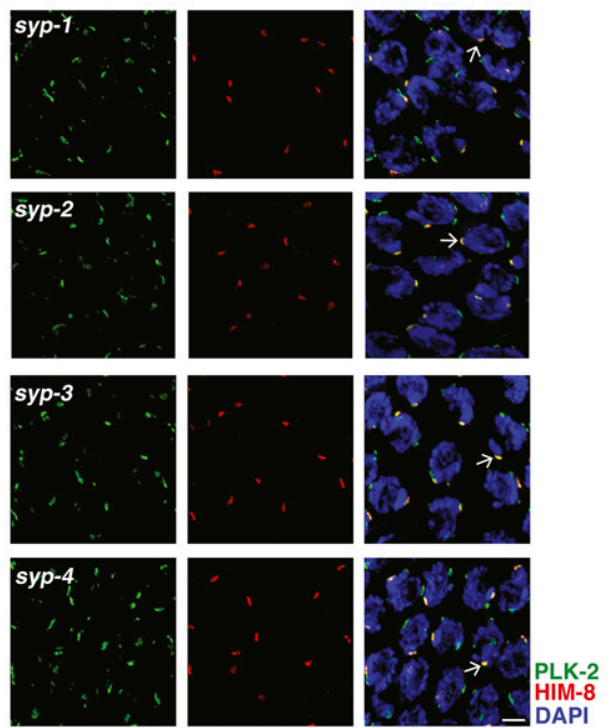

Figure 5 syp-3 mutants have active PCs. (A) Images of germlines, from entry into meiosis until late meiotic prophase, of wild-type worms, syp-1, syp-2, syp-3, and syp-4 mutants stained to visualize PLK-2 (green and grayscale) and DAPI (red). Delay in meiotic progression indicated by white dashed line. Bar, $30 \mu \mathrm{m}$. (B) Images of early meiotic prophase nuclei in wild-type worms, syp-1, syp-2, syp-3, and syp-4 mutants stained to visualize ZIM-2 (yellow) and DAPI (blue). (C) Images of early meiotic prophase nuclei in wild-type worms, syp-1, syp-2, syp-3, and syp-4 mutants stained to visualize PLK-2 (green), HIM-8 (red), and DAPI (blue). Arrow indicates an example of colocalization of PLK-2 and HIM-8. Bar, $2 \mu \mathrm{m}$.

mutants in early meiotic prophase nuclei (Figure 4A). In wild-type worms ZIM-2 forms robust patches at the nuclear periphery (Figure 4A) (Phillips and Dernburg 2006). We observed ZIM-2 staining in $h t p-1$ htp-2 double mutants similar to wild-type worms (Figure 4A). However, $h t p-3$ and him-3 mutants had less robust ZIM-2 localization compared to wildtype worms (Figure 4A). We saw similar results in $h$ tp-3, him3 , and $h t p-1 h t p-2$ mutants when we stained for ZIM-1 and ZIM-3 (Supplemental Material, Figure S1, A and B), which bind the PCs of chromosomes I and IV and chromosomes II and III, respectively (Phillips and Dernburg 2006).

The defect in robustly localizing ZIMs to PCs in $h t p-3$ and him-3 mutants (Figure 4A and Figure S1, A and B) might explain why these mutants are defective in the synapsis checkpoint. However, a single unsynapsed $X$ chromosome, with an active PC, is sufficient to elicit a checkpoint response (Bhalla and Dernburg 2005). Therefore, we also localized the $X$ chromosome PC binding protein, HIM-8 (Figure 4B) (Phillips et al. 2005). We observed staining patterns similar to wild-type worms in htp-3, him-3, and htp-1 htp-2 mutants (Figure 4B). However, consistent with published reports (Couteau et al. 2004; Couteau and Zetka 2005; Goodyer et al. 2008), HIM-8 foci were more often unpaired in $h t p-3$ and him-3 mutants, while in wild-type and $h t p-1 h t p-2$ double mutants a single HIM-8 focus per nucleus could often be observed in early meiotic prophase nuclei. We also determined whether $X$ chromosome PCs were functional in these mutant backgrounds by localizing PLK-2 (Figure 4B), a kinase that is recruited by PCs to promote synapsis and the synapsis checkpoint (Harper et al. 2011; Labella et al. 2011). In htp-3, him-3, and htp-1 htp-2 mutants, PLK-2 colocalized with HIM-8 (Figure 4B), indicating $X$ chromosome PCs were active. Altogether, these data argue against the interpretation that mutations in HORMAD proteins abrogate the synapsis checkpoint indirectly due to defects in PC function and support the conclusion that they are involved in the synapsis checkpoint response.

\section{syp-3 mutants have active PCs}

Similar to htp-3, him-3, and htp-1 htp-2 mutants, syp-3 mutants have unsynapsed chromosomes but fail to elevate germline apoptosis in response to the synapsis checkpoint (Figure 1D). Unlike $h t p-3$, him-3, and htp-1 htp-2 mutants, syp-3 mutants display a delay in meiotic progression (Smolikov et al. 


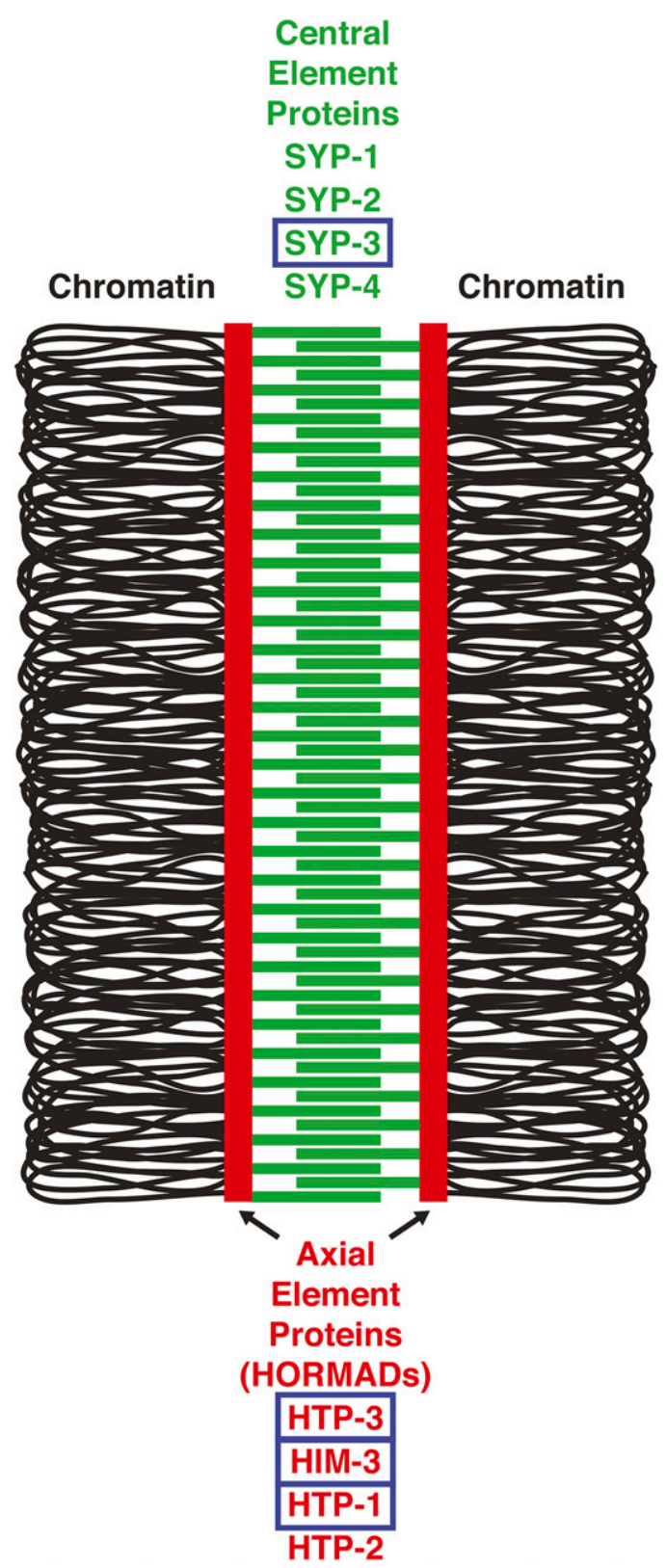

Figure 6 Cartoon of the SC in C. elegans. Central element components are in green (SYP-1, SYP-2, SYP-3, and SYP-4) and axial element components (HORMADs) are in red (HTP-3, HIM-3, HTP-1, and HTP-2). Chromatin is depicted as black loops tethered by axial elements. SC components that are required for the synapsis checkpoint are boxed in blue.

2007), likely because HTP-3, HIM-3, HTP-1, and HTP-2 are present to promote this delay (Martinez-Perez and Villeneuve 2005; Kim et al. 2015). However, this delay in meiotic progression does not depend on PC function (Kim et al. 2015), raising the possibility that syp-3 mutants abrogate the synapsis checkpoint due to defective PCs. To directly test this, we localized PLK-2 in meiotic prophase in syp-3 mutants and compared them to wild-type worms, syp-1, syp-2, and syp-4 mutants. Similar to wild-type animals and syp-1 (Harper et al. 2011; Labella et al. 2011), syp-2, and syp-4 mutants, syp-3 mutants robustly localized PLK-2 to PCs (Figure 5A). Moreover, unlike wild-type germlines, PLK-2 localization on PCs is extended in syp-3 mutants, similar to syp-1, syp-2, and syp-4 mutants (Figure $5 \mathrm{~A}$ ).

We complemented this evaluation of PC function by localizing ZIM-2 and HIM- 8 in syp-3 mutants and compared this to syp-1, syp-2, and syp-4 mutants. ZIM-2 forms robust patches in meiotic nuclei in syp-3 mutants, similar to syp-1, -2 , and -4 mutants (Figure 5B). Furthermore, HIM-8 localizes to all meiotic nuclei in syp-3 mutants and colocalizes with PLK-2 (Figure 5C). These data show that SYP-3 is required for the synapsis checkpoint in a mechanism distinct from regulating PC function.

Altogether, our data demonstrate that some SC components, namely SYP-3, HTP-3, HIM-3, and HTP-1, are required for the synapsis checkpoint (Figure 6). Furthermore, their involvement in the synapsis checkpoint does not correlate with their effects on PC pairing (Figure 2 and Couteau et al. 2004; Couteau and Zetka 2005; Goodyer et al. 2008), suggesting they contribute to synapsis checkpoint function in some unique fashion. We propose that the synapsis checkpoint monitors SC assembly via these SC components. Uncovering which specific functions of SYP-3 and the HORMADs are required for the synapsis checkpoint are intriguing questions to be addressed in future studies.

Surprisingly, despite having similar defects in synapsis, we found that not all central element components of the SC are equivalent in the context of checkpoint function. While syp-2 mutants essentially phenocopy syp- 1 mutants, syp-4 mutants have a functional synapsis checkpoint that is independent of PCH-2 but dependent on MDF-1. When combined with our pairing analysis (Figure 2B), these data raise the possibility that SYP-4 could be playing another role during the synapsis checkpoint. SYP-4 was identified by virtue of its two-hybrid interaction with SYP-3. However, unlike SYP-3, SYP-4 does not show an interaction with either SYP-1 or SYP-2 by twohybrid assay (Smolikov et al. 2009). While there are a variety of reasons why relevant protein-protein interactions might not be recapitulated by yeast two-hybrid assays, these negative data suggest that SYP-4 could uniquely interact with SYP-3 during synapsis. For example, one scenario consistent with our data is that when SYP-3 is not bound to SYP-4, SYP-3 signals to the synapsis checkpoint and when it is bound to SYP-4, this signal is silenced. Future experiments will address this hypothesis.

\section{Materials and Methods}

\section{Genetics and Worm Strains}

The wild-type C. elegans strain background was Bristol N2 (Brenner 1974). All experiments were performed on adult hermaphrodites at $20^{\circ}$ under standard conditions. Mutations and rearrangements used were as follows:

LG I: htp-3(tm3655), syp-4 (tm2713), cep-1 (gk138), syp-3(ok258), hT2 [bli-4(e937) let-?(q782) qIs48] (I, III). LG II: pch-2(tm1458). 
LG IV: htp-1 (gk174), htp-2(tm2543), him-3(gk149), spo11(ok79), nT1 [unc-?(n754) let-?(m435)] (IV, V), nT1 [qIs51] (IV, V).

LG V: syp-2(ok307), syp-1(me17), mdf-1(av19), bcIs39(Pim:: ced-1::GFP).

\section{Quantification of germline apoptosis}

Scoring of germline apoptosis was performed as previously described in Bhalla and Dernburg (2005). L4 hermaphrodites were allowed to age for $22 \mathrm{hr}$ at $20^{\circ}$. Live worms were mounted under coverslips on $1.5 \%$ agarose pads containing $0.2 \mathrm{mM}$ levamisole. A minimum of 25 germlines were analyzed for each genotype by performing live fluorescence microscopy and counting the number of cells fully surrounded by CED-1::GFP. Significance was assessed using a paired t-test between all mutant combinations. All experiments were performed at least twice.

\section{Antibodies, immunostaining, and microscopy}

Immunostaining was performed on worms 20- to 24-hr postL4 stage. Gonad dissections were carried out in $1 \times \mathrm{EBT}$ (250 mM HEPES-Cl, pH 7.4, $1.18 \mathrm{M} \mathrm{NaCl}, 480 \mathrm{mM} \mathrm{KCl,}$ $20 \mathrm{mM}$ EDTA, $5 \mathrm{mM}$ EGTA) + 0.1\% Tween 20 and $20 \mathrm{mM}$ sodium azide. An equal volume of $2 \%$ formaldehyde in EBT (final concentration was 1\% formaldehyde) was added and allowed to incubate under a coverslip for $5 \mathrm{~min}$. The sample was mounted on HistoBond $(75 \times 25 \times 1 \mathrm{~mm}$ from VWR) slides and freeze cracked and incubated in methanol at $-20^{\circ}$ for $1 \mathrm{~min}$ and transferred to PBS with Tween 20 (PBST). Following several washes of PBST, the samples were incubated for $30 \mathrm{~min}$ in $1 \%$ bovine serum albumin diluted in PBST. A hand-cut paraffin square was used to cover the tissue with $50 \mu$ l of antibody solution. Incubation was conducted in a humid chamber overnight at $4^{\circ}$. Slides were rinsed in PBST, then incubated for $2 \mathrm{hr}$ at room temperature with fluorophore-conjugated secondary antibody at a dilution of 1:500. The samples were then mounted in $13 \mu \mathrm{l}$ of mounting media [20 M N-propyl gallate (Sigma, St. Louis, MO) and $0.14 \mathrm{M}$ Tris in glycerol) with a no. $1.5\left(22 \mathrm{~mm}^{2}\right)$ coverslip and sealed with nail polish.

Primary antibodies were as follows (dilutions are indicated in parentheses): guinea pig anti-ZIM-2 (1:2500; Phillips and Dernburg 2006), guinea pig anti-PLK-2 (1:750; Harper et al. 2011), and rat anti-HIM-8 (1:250; Phillips and Dernburg 2006). Secondary antibodies were as follows: Cy3 anti-rabbit (Jackson Immunochemicals, West Grove, PA) and Alexa Fluor-488 anti-guinea pig and anti-rat (Invitrogen, Carlsbad, CA).

Quantification of pairing was performed with a minimum of three whole germlines per genotype as in Phillips et al. (2005) on animals 24 hr post-L4 stage.

All images were acquired at room temperature using a DeltaVision Personal DV system (Applied Precision) equipped with a $100 \times$ N.A. 1.40 oil-immersion objective (Olympus), resulting in an effective XY pixel spacing of 0.064 or $0.040 \mu \mathrm{m}$. Images were captured using a "camera" threedimensional image stacks were collected at $0.2-\mu \mathrm{m} Z$-spacing and processed by constrained, iterative deconvolution. Imaging, image scaling, and analysis were performed using functions in the softWoRx software package. Projections were calculated by a maximum intensity algorithm. Composite images were assembled and some false coloring was performed with Adobe Photoshop.

\section{Acknowledgments}

We thank Abby Dernburg and Anne Villeneuve for valuable strains and reagents. This work was supported by the National Institutes of Health (NIH) [T32-GM008646 (to T.B.) and R01-GM097144 (to N.B.)] and a DeAntonio Summer Undergraduate research award (to G.A.). Some strains were provided by the Caenorhabditis Genetics Center, which is funded by the NIH Office of Research Infrastructure Programs (P40-OD010440). The authors declare no competing financial interests.

\section{Literature Cited}

Aravind, L., and E. V. Koonin, 1998 The HORMA domain: a common structural denominator in mitotic checkpoints, chromosome synapsis and DNA repair. Trends Biochem. Sci. 23: 284-286.

Bhalla, N., and A. F. Dernburg, 2005 A conserved checkpoint monitors meiotic chromosome synapsis in Caenorhabditis elegans. Science 310: 1683-1686.

Bhalla, N., and A. F. Dernburg, 2008 Prelude to a division. Annu. Rev. Cell Dev. Biol. 24: 397-424.

Bhalla, N., D. J. Wynne, V. Jantsch, and A. F. Dernburg, 2008 ZHP-3 acts at crossovers to couple meiotic recombination with synaptonemal complex disassembly and bivalent formation in C. elegans. PLoS Genet. 4: e1000235.

Bohr, T., C. R. Nelson, E. Klee, and N. Bhalla, 2015 Spindle assembly checkpoint proteins regulate and monitor meiotic synapsis in C. elegans. J. Cell Biol. 211: 233-242.

Brenner, S., 1974 The genetics of Caenorhabditis elegans. Genetics 77: 71-94.

Carballo, J. A., A. L. Johnson, S. G. Sedgwick, and R. S. Cha, 2008 Phosphorylation of the axial element protein Hop1 by Mec1/Tel1 ensures meiotic interhomolog recombination. Cell 132: 758-770.

Caryl, A. P., S. J. Armstrong, G. H. Jones, and F. C. Franklin, 2000 A homologue of the yeast HOP1 gene is inactivated in the Arabidopsis meiotic mutant asy1. Chromosoma 109: 62-71.

Colaiacovo, M. P., A. J. MacQueen, E. Martinez-Perez, K. McDonald, A. Adamo et al., 2003 Synaptonemal complex assembly in C. elegans is dispensable for loading strand-exchange proteins but critical for proper completion of recombination. Dev. Cell 5: 463-474.

Couteau, F., K. Nabeshima, A. Villeneuve, and M. Zetka, 2004 A component of C. elegans meiotic chromosome axes at the interface of homolog alignment, synapsis, nuclear reorganization, and recombination. Curr. Biol. 14: 585-592.

Couteau, F., and M. Zetka, 2005 HTP-1 coordinates synaptonemal complex assembly with homolog alignment during meiosis in C. elegans. Genes Dev. 19: 2744-2756. 
Daniel, K., J. Lange, K. Hached, J. Fu, K. Anastassiadis et al., 2011 Meiotic homologue alignment and its quality surveillance are controlled by mouse HORMAD1. Nat. Cell Biol. 13: 599-610.

Dernburg, A. F., K. McDonald, G. Moulder, R. Barstead, M. Dresser et al., 1998 Meiotic recombination in C. elegans initiates by a conserved mechanism and is dispensable for homologous chromosome synapsis. Cell 94: 387-398.

Derry, W. B., A. P. Putzke, and J. H. Rothman, 2001 Caenorhabditis elegans p53: role in apoptosis, meiosis, and stress resistance. Science 294: 591-595.

Deshong, A. J., A. L. Ye, P. Lamelza, and N. Bhalla, 2014 A quality control mechanism coordinates meiotic prophase events to promote crossover assurance. PLoS Genet. 10: e1004291.

Fukuda, T., K. Daniel, L. Wojtasz, A. Toth, and C. Hoog, 2010 A novel mammalian HORMA domain-containing protein, HORMAD1, preferentially associates with unsynapsed meiotic chromosomes. Exp. Cell Res. 316: 158-171.

Goodyer, W., S. Kaitna, F. Couteau, J. D. Ward, S. J. Boulton et al., 2008 HTP-3 links DSB formation with homolog pairing and crossing over during C. elegans meiosis. Dev. Cell 14: 263-274.

Harper, N. C., R. Rillo, S. Jover-Gil, Z. J. Assaf, N. Bhalla et al., 2011 Pairing centers recruit a Polo-like kinase to orchestrate meiotic chromosome dynamics in C. elegans. Dev. Cell 21: 934 947.

Hassold, T., and P. Hunt, 2001 To err (meiotically) is human: the genesis of human aneuploidy. Nat. Rev. Genet. 2: 280-291.

Hollingsworth, N. M., L. Goetsch, and B. Byers, 1990 The HOP1 gene encodes a meiosis-specific component of yeast chromosomes. Cell 61: 73-84.

Kim, Y., S. C. Rosenberg, C. L. Kugel, N. Kostow, O. Rog et al., 2014 The chromosome axis controls meiotic events through a hierarchical assembly of HORMA domain proteins. Dev. Cell 31: 487-502.

Kim, Y., N. Kostow, and A. F. Dernburg, 2015 The chromosome axis mediates feedback control of CHK-2 to ensure crossover formation in C. elegans. Dev. Cell 35: 247-261.

Labella, S., A. Woglar, V. Jantsch, and M. Zetka, 2011 Polo kinases establish links between meiotic chromosomes and cytoskeletal forces essential for homolog pairing. Dev. Cell 21: 948-958.

MacQueen, A. J., and A. Hochwagen, 2011 Checkpoint mechanisms: the puppet masters of meiotic prophase. Trends Cell Biol. 21: 393-400.

MacQueen, A. J., M. P. Colaiacovo, K. McDonald, and A. M. Villeneuve, 2002 Synapsis-dependent and -independent mechanisms stabilize homolog pairing during meiotic prophase in C. elegans. Genes Dev. 16: 2428-2442.

MacQueen, A. J., C. M. Phillips, N. Bhalla, P. Weiser, A. M. Villeneuve et al., 2005 Chromosome sites play dual roles to establish homologous synapsis during meiosis in C. elegans. Cell 123: 1037-1050.
Martinez-Perez, E., and A. M. Villeneuve, 2005 HTP-1-dependent constraints coordinate homolog pairing and synapsis and promote chiasma formation during C. elegans meiosis. Genes Dev. 19: $2727-2743$.

Phillips, C. M., and A. F. Dernburg, 2006 A family of zinc-finger proteins is required for chromosome-specific pairing and synapsis during meiosis in C. elegans. Dev. Cell 11: 817-829.

Phillips, C. M., C. Wong, N. Bhalla, P. M. Carlton, P. Weiser et al., 2005 HIM-8 binds to the $\mathrm{X}$ chromosome pairing center and mediates chromosome-specific meiotic synapsis. Cell 123: 1051-1063.

Rosu, S., K. A. Zawadzki, E. L. Stamper, D. E. Libuda, A. L. Reese et al., 2013 The C. elegans DSB-2 protein reveals a regulatory network that controls competence for meiotic DSB formation and promotes crossover assurance. PLoS Genet. 9: e1003674.

Schumacher, B., K. Hofmann, S. Boulton, and A. Gartner, 2001 The C. elegans homolog of the p53 tumor suppressor is required for DNA damage-induced apoptosis. Curr. Biol. 11: 1722-1727.

Smolikov, S., A. Eizinger, K. Schild-Prufert, A. Hurlburt, K. McDonald et al., 2007 SYP-3 restricts synaptonemal complex assembly to bridge paired chromosome axes during meiosis in Caenorhabditis elegans. Genetics 176: 2015-2025.

Smolikov, S., K. Schild-Prufert, and M. P. Colaiacovo, 2009 A yeast two-hybrid screen for SYP-3 interactors identifies SYP-4, a component required for synaptonemal complex assembly and chiasma formation in Caenorhabditis elegans meiosis. PLoS Genet. 5: e1000669.

Stamper, E. L., S. E. Rodenbusch, S. Rosu, J. Ahringer, A. M. Villeneuve et al., 2013 Identification of DSB-1, a protein required for initiation of meiotic recombination in Caenorhabditis elegans, illuminates a crossover assurance checkpoint. PLoS Genet. 9: e1003679.

Woglar, A., A. Daryabeigi, A. Adamo, C. Habacher, T. Machacek et al., 2013 Matefin/SUN-1 phosphorylation is part of a surveillance mechanism to coordinate chromosome synapsis and recombination with meiotic progression and chromosome movement. PLoS Genet. 9: e1003335.

Wojtasz, L., K. Daniel, I. Roig, E. Bolcun-Filas, H. Xu et al., 2009 Mouse HORMAD1 and HORMAD2, two conserved meiotic chromosomal proteins, are depleted from synapsed chromosome axes with the help of TRIP13 AAA-ATPase. PLoS Genet. 5: e1000702.

Wojtasz, L., J. M. Cloutier, M. Baumann, K. Daniel, J. Varga et al., 2012 Meiotic DNA double-strand breaks and chromosome asynapsis in mice are monitored by distinct HORMAD2-independent and -dependent mechanisms. Genes Dev. 26: 958-973.

Zetka, M. C., I. Kawasaki, S. Strome, and F. Muller, 1999 Synapsis and chiasma formation in Caenorhabditis elegans require HIM-3, a meiotic chromosome core component that functions in chromosome segregation. Genes Dev. 13: 2258-2270.

Communicating editor: N. Hunter 
